affective disorder in old age, probably with causality in both directions (Phillpott, 1990). Clearly physical ill health is an important factor in the elderly suffering with dementia.

The very high degree of physical morbidity was confirmed in a survey carried out by myself on in-patients in an acute functional and an acute dementia unit for the elderly which included 19 patients on the dementia ward and 22 patients on the functional ward. On the dementia ward each patient was on a mean of 4.8 medications of which 3.4 medications were prescribed for physical disorder. On the functional ward each patient was on a mean of $\mathbf{5 . 2}$ medications of which 2.9 were prescribed for physical disorder. On the dementia ward ten out of the 19 patients had an acute physical illness (as defined by onset within two weeks). Also in the dementia group a total of 59 chronic illnesses were detected (on average 3.1 chronic conditions per patient). There were no acute physical illnesses detected on the functional ward but there were 42 chronic physical conditions (an average of 1.9 chronic conditions per patient).

In view of these findings it is interesting to note that old age psychiatry wards are often staffed by registered mental nurses and psychiatric trainees. Generally a medical opinion may only be obtained by specific referral rather than regular liaison attendance or joint psychogeriatric- geriatric assessments. Junior medical staff, often in isolated psychiatric institutions, do have difficulty obtaining urgent medical opinions and may also meet a degree of prejudice. The management of physical ill health in the psychiatric setting is one that requires further investigation.

PHulport, R. (1990) Affective disorder and physical illness. International Clinical Psychopharmacology, B, Supplement 3.

S.F. Foster, Registrar in Psychiatry, Mersey Regional Rotation

\section{A study of the use of routine laboratory investigations in adult psychiatric patients}

Sir: The inappropriate use of laboratory tests observed in clinical practice (Sandler, 1979) appears to be a problem in psychiatric practice as well (White \& Barraclough, 1989).

We undertook a questionnaire study of adult psychiatric patients admitted to St Lukes Hospital, Middlesbrough during a three month period in 1992 to examine the use of laboratory tests and establish the pattern of physical testing in psychiatric practice. Of the 119 patients studied during this period, 43 were transferred or discharged without any diagnostic tests performed. Thirty-eight different tests were carried out on the remaining patients with a range of 1-12 tests per patient. Full blood count (66 tests) and biochemistry (SMAC) (74) were the most frequently requested tests. Thyroid functions tests (37), urine examination (13) and serum lithium levels (22) were also performed. Other physical tests were electroencephalograms (19), electrocardiograms (11), X-rays (8), serum drug levels in selfpoisoning (7).

The positive rate for full blood count was $18 \%$, biochemistry (SMAC) 24\%, thyroid function tests $13 \%$, urine $8 \%$ and serum lithium $32 \%$. More tests ( 3 tests/patient) were performed on patients who were older (46-65 years) and were in-patients (5 tests/patient) for a longer period ( 29 days or more). No significant differences were detected between males and females or between diagnostic groups.

The helpfulness of the tests was assessed by examining the clinical details in the patients' records; $80 \%$ of blochemistry tests were helpful, $57 \%$ of full blood counts, $100 \%$ of thyroid function tests and $79 \%$ of urine examinations. The reasons given for assessing the helpfulness of the tests were: exclusion of physical causes (23), estimation of therapeutic level (17), assessment of liver function (8) and adjustment of dosage of drugs (4).

This study demonstrates that laboratory tests are performed very frequently on psychiatric patients as a matter of practice and the pattern appears to be similar to that in other psychiatric hospitals and general medical wards. Training programmes for medical staff, protocols and guidelines, reviews and audit may help to minimise the inappropriateness of these tests.

SANDLER, G. (1979) Costs of unnecessary tests. British Medical Journal, u, 21-24.

WhITE, A.J. \& BARRACLOUGH, B. (1989) Benefits and problems of routine laboratory investigations in adult psychiatric admissions. Brittsh Journal of Psychiatry. 185, 65-72.

K.P. RAO, St Luke's Hospttal, Middlesbrough, Cleveland and R.S. RAMAIAH, Public Health Medicine, Walsall Health Authority, Walsall, West Midlands

\section{Psychiatrists and euthanasia}

Sir: Dr Huyse and colleagues have provided us with an interesting paper on 'Consultant-liaison psychiatrists and euthanasia in The Netherlands' (Psychiatric Bulletin, August 1994, 18, 497-500). However, euthanasia is not practised in most countries represented by the wide readership of the Bulletin. Indeed in the UK readers may be interested to know that the government has just accepted the recommendations of the House of Lords Medical Ethics Committee not to legalise euthanasia.

M. DOMINIC BEER, UMDS Guy's and Bexley Hospital, Bexley, Kent DA5 2BW 